\title{
Oxymatrine induces A549 human non-small lung cancer cell apoptosis via extrinsic and intrinsic pathways
}

\author{
GUANG-ZHOU ZHOU ${ }^{1}$, YAN-YAN SHI ${ }^{1}$, LIU-SU CUI ${ }^{2}$, A-FANG LI ${ }^{1}$, QING-QING WANG ${ }^{1}$ and MIN LIU $^{3}$ \\ ${ }^{1}$ Department of Biotechnology, College of Bioengineering, Henan University of Technology, Zhengzhou, Henan 450001; \\ ${ }^{2}$ Laboratory of Morphology, College of Basic Medical Sciences, Xinxiang Medical University, Xinxiang, Henan 453003; \\ ${ }^{3}$ Department of Infectious Diseases, The Xiang'an Hospital of Xiamen University, Xiamen, Fujian 361005, P.R. China
}

Received April 20, 2017; Accepted September 11, 2017

DOI: $10.3892 / \mathrm{mmr} .2017 .7982$

\begin{abstract}
Oxymatrine is one of the primary natural compounds extracted from the Sophora flavescens, and has been reported to exhibit numerous pharmacological properties including cancer-preventive and anti-cancer effects, however the mechanisms as to how oxymatrine exhibits anti-proliferative activity in non-small cell lung carcinoma cells remains uncertain. The present study aimed to explore the mechanism of its anti-cancer effect, and whether it is due to apoptosis induction and anti-migration in the A549 lung cancer cell line. Detection of morphological alterations, MTT analysis, Hoechst/propidium iodide dual staining and terminal deoxynucleotidyl transferase dUTP nick end labeling assays verified that oxymatrine induced A549 cell apoptosis. The caspase pan-inhibitor z-VAD-FMK resulted in disappearance of oxymatrine-elicited nuclei fragmentation via Hoechst 33342 staining. JC-1 staining demonstrated a decrease in mitochondrial membrane potential which further verified the induction of apoptosis by oxymatrine. The caspase-3, 8 and 9 activities of oxymatrine-treated cells were activated, which suggested that extrinsic and intrinsic apoptotic pathways were involved in the anti-proliferative effects of oxymatrine in A549 cells. Furthermore, the wound healing assay verified the anti-migratory effects of oxymatrine in A549 cells.
\end{abstract}

\section{Introduction}

As one of the most active components of the ethanol extracts from Sophoraflavescens, oxymatrine (OMT) has been of primary research interest, in view of its biological activities and wide range of pharmacological effects, including

Correspondence to: Dr Guang-Zhou Zhou, Department of Biotechnology, College of Bioengineering, Henan University of Technology, 100 Lianhua Street, Zhengzhou, Henan 450001, P.R. China

E-mail: gzzhou@163.com

Key words: oxymatrine, cell apoptosis, A549 lung cancer cells, anti-proliferation anti-inflammatory, anti-allergic, anti-fibrotic and cardiovascular protective activities $(1,2)$. In particular, it has been extensively used for the treatment of chronic hepatitis $\mathrm{B}$, and exhibited high clinical value (3). Previously, oxymatrine has demonstrated an anti-tumor cell proliferative effect, and synergistically enhances anti-tumor activity of oxaliplatin in colon carcinoma (4). Li et al (5) revealed that oxymatrine suppresses proliferation and facilitates apoptosis of human ovarian cancer cells, through upregulating microRNA-29b and downregulating matrix metallopeptidase-2 expression levels. The effects of oxymatrine on the proliferation and apoptosis of SMMC-7721 human hepatoma carcinoma cells have additionally been investigated, and have been proven to exhibit a therapeutic advantage for future chemoprevention or chemotherapy (6). Anti-proliferative abilities of oxymatrine have additionally been observed in prostate cancer, gastric cancer and leukemia cells $(1,7,8)$, and subsequently introduced oxymatrine as a potential novel therapeutic in the prevention and treatment of cancer (1).

As one leading cause of cancer-associated mortality globally, lung cancer predominantly comprises small-cell lung carcinoma (SCLC) and non-small cell lung carcinoma (NSCLC), which account for 80-85 and 15-20\% of all lung cancer cases, respectively $(9,10)$. Despite scientific progress, patients exhibiting NSCLC still experience short survival times and high mortality (11). Conventional treatments of lung cancer, including platinum-based chemotherapy and/or radiation therapy are fairly ineffective, and result in a host of negative side effects (12). As a result, the requirement for the discovery of alternative novel drugs and less toxic therapeutic strategies for the treatment of NSCLC is evident. Previous studies have suggested that numerous natural extracts and chemically synthesized compounds exhibit therapeutic efficacy as potential candidates $(13,14)$. The authors previously identified several curcumin analogs with anti-proliferative effects on A549 lung cancer cells and studied their molecular mechanisms of apoptosis, induced by the MHMD [(1E,6E)-4-(furan2yl) methylene]-1,7-bis(4-hydroxy-3-methoxyphe-nyl) hepta-1, 6-diene-3,5-dione), IHCH(2E,6E-2-(1H-indol-3-yl) methylene)-6-(4-hydroxy-3-methoxybenzylidene)-cyclohexanone) and HBC (hydrazinobenzoylcurcumin), which represent possible therapeutic agents (15-17).

In the present study, the compound oxymatrine was applied to inhibit proliferation of A549 cells. It was demonstrated 
that oxymatrine induced apoptosis of A549 cells using Hoechst/propidium iodide (PI) staining, terminal deoxynucleotidyl transferase dUTP nick end labeling assays (TUNEL) analysis and caspase inhibition experiments. Furthermore, caspases-3, 8 and 9 of A549 cells were revealed to be activated following treatment with oxymatrine, which suggested that caspase-dependent extrinsic and intrinsic mitochondrial pathways of apoptosis were involved in the anti-proliferative effects of oxymatrine. The present study aimed to elucidate the anti-proliferative molecular mechanism of oxymatrine in lung cancer cells, and provide a potential candidate to act as a future antitumor agent.

\section{Materials and methods}

Chemicals and cell culture. Oxymatrine was a kind gift from Professor Min Liu, (The Xiang'an Hospital of Xiamen University, Xiamen, China). It was dissolved in PBS solution (stock concentration of $100 \mathrm{mg} / \mathrm{ml}$ ). RPMI-1640 medium and newborn calf serum (NCS; Gibco, Thermo Fisher Scientific, Inc., Waltham, MA, USA) were purchased from Beijing DingguoChangsheng Biotechnology, Co. Ltd. (Beijing, China). A549 cells were cultured in 1640 medium supplemented with $10 \%$ calf serum and $1 \%$ garamycin at $37^{\circ} \mathrm{C}$ in an incubator in an environment containing $5 \% \mathrm{CO}_{2}$.

Cell proliferation assay of oxymatrine. A549 cells were seeded into 12-well culture plates (Corning Incorporated, Corning, NY, USA) at a density of $1 \times 10^{5}$ cells per well by using Blood Counting chambers, that would allow the formation of a $90 \%$ monolayer following $24 \mathrm{~h}$. A total of six different final concentrations of oxymatrine $(0.1,0.5,1.0,1.5,2.0$ and $2.5 \mathrm{mg} / \mathrm{ml})$ were determined and added into the wells. The treated cells were then incubated at $37^{\circ} \mathrm{C}$ for different time periods. Pictures were taken by light microscope to detect cell morphological alterations at different time points in oxymatrine-treated A549 cells.

Cell viability assay. A549 cells were incubated in 96-well plates $\left(1 \times 10^{4}\right.$ cells/well) in an incubator containing $5 \%$ $\mathrm{CO}_{2}$ at $37^{\circ} \mathrm{C}$. Then, cells were treated with oxymatrine at different final concentrations for $24 \mathrm{~h}$. A total of $20 \mu \mathrm{l}$ MTT [3-(4,5-dimethylthiazolyl-2)-2,5-diphenyltetrazolium bromide, stock $0.1 \mathrm{M}$ ] was added into each well and cells were placed in the dark for $4 \mathrm{~h}$ at $37^{\circ} \mathrm{C}$. Following aspiration of supernatant, dimethyl sulfoxide (100 $\mu \mathrm{l} /$ well) was added to the wells, and cells were kept in the dark at room temperature, on a mixer for $30 \mathrm{~min}$. The absorbance $\left(\mathrm{OD}_{570}\right)$ was measured at a wavelength of $495 \mathrm{~nm}$ by a microplate spectrophotometer (BioTek China, Beijing, China) to determine cell viability. All the experiments were repeated in triplicate. $\mathrm{P}<0.05$ and $\mathrm{P}<0.01$ were considered to indicate a statistically significant difference.

Fluorescence microscopy analysis. A549 cells were incubated in 6 -well plates $\left(3 \times 10^{5}\right.$ cells/well) and incubated in an environment containing $5 \% \mathrm{CO}_{2}$. Oxymatrine $(1.5 \mathrm{mg} / \mathrm{ml})$ was used to treat cells in exponential growth period for 24,48 and $72 \mathrm{~h}$, respectively. Following immobilization with $4 \%$ paraformaldehyde for $10 \mathrm{~min}$ at room temperature, A549 cells were stained with Hoechest $33342(10 \mu \mathrm{g} / \mathrm{ml})$ and PI, $(10 \mu \mathrm{g} / \mathrm{ml})$ for $10 \mathrm{~min}$ in $37^{\circ} \mathrm{C}$ incubator successively. Following washing three times with PBS, the treated cells were sealed with glycerol $(50 \%$ in PBS) and then observed under the fluorescence microscope (Nikon TE2000-U or Nikon 80i; Nikon Corporation, Tokyo, Japan).

For the mitochondrial membrane potential assay, the green JC-1 (GenView SA, Lausanne, Switzerland) signals were measured at Ex $485 \mathrm{~nm} / \mathrm{Em} 528 \mathrm{~nm}$, whereas the red signals were detected at Ex $540 \mathrm{~nm} / \mathrm{Em} 590 \mathrm{~nm}$. The absorbance was determined by a microplate spectrophotometer (BioTek China).

For caspase inhibition analysis, A549 cells were firstly incubated with $50 \mu \mathrm{M}$ z-VAD-FMK (Beyotime Institute of Biotechnology, Haimen, China) for $30 \mathrm{~min}$. Then, the A549 cells were treated with oxymatrine $(1.5 \mathrm{mg} / \mathrm{ml})$ for another $48 \mathrm{~h}$. Finally, the fluorescence microscope analysis was carried out in accordance with the aforementioned procedure.

TUNEL analysis. A549 cells ( $3 \times 10^{5}$ cells/well) were cultured under the aforementioned conditions, and oxymatrine (final concentration of $1.5 \mathrm{mg} / \mathrm{ml}$ ) was incubated with cells for $48 \mathrm{~h}$. Then, apoptotic cells were analyzed according to the TUNEL kit manufacturer's protocol (DeadEND ${ }^{\mathrm{TM}}$ Colorimetric Apoptosis Detection System; Promega Corporation, Madison, WI, USA).

Flow cytometry analysis. A549 cells $\left(3 \times 10^{5}\right.$ cells $\left./ \mathrm{ml}\right)$ were seeded into a $30-\mathrm{mm}$ cell culture dish and treated with oxymatrine $(1 \mathrm{mM})$ for 24,48 and $72 \mathrm{~h}$, respectively. The A549 cells were fixed (70\% ethanol) for $2 \mathrm{~h}$ at $4^{\circ} \mathrm{C}$, washed with PBS, permeabilized $(0.1 \%$ Triton X-100) and stained (PI-FITC) in accordance with the procedure described in Zhou et al (17). Then, cell samples were employed for the flow cytometry assay. Data were collected and analyzed by the CellQuest Prosoftware (version 6.0; FACS Calibur; BD Biosciences, Franklin Lakes, NJ, USA).

Caspase activity detection. Mock-treated or oxymatrine-treated $(1.5 \mathrm{mg} / \mathrm{ml})$ A549 cells $(48 \mathrm{~h})$ were harvested by scraping, washed with PBS, frozen for $30 \mathrm{~min}$ and centrifuged $\left(16,000 \mathrm{x}\right.$ g for $15 \mathrm{~min}$ at $\left.4^{\circ} \mathrm{C}\right)$. The supernatants were assayed by a plate reader (Model 550 Microplate Reader; Bio-Rad Laboratories, Inc., Hercules, CA) at a wavelength of $405 \mathrm{~nm}$, for the activities of caspase-3, 8, 9 in the presence of $20 \mu \mathrm{M}$ Ac-DEVD-pNA, Ac-IETD-pNA and Ac-LEHD-pNA (Beyotime Institute of Biotechnology, Haimen, China), respectively. The experiments were carried out in triplicate.

Wound healing assay. The migratory activity of A549 cells was determined using a wound healing assay, as previously described (17). Briefly, one pipette tip was used to make a scratch wound across each well in a 12 -well plate. Following washing and removing any loosely held cells, oxymatrine $(1.5 \mathrm{mg} / \mathrm{ml})$ was added into each well for 24,48 and $72 \mathrm{~h}$, respectively. An inverted microscope was used to track the migration process of A549 cells.

Statistical analysis. One-way analysis of variance followed by LSD and Tukey post-hoc tests was used to evaluate differences between groups. All data were analyzed by SPSS software 

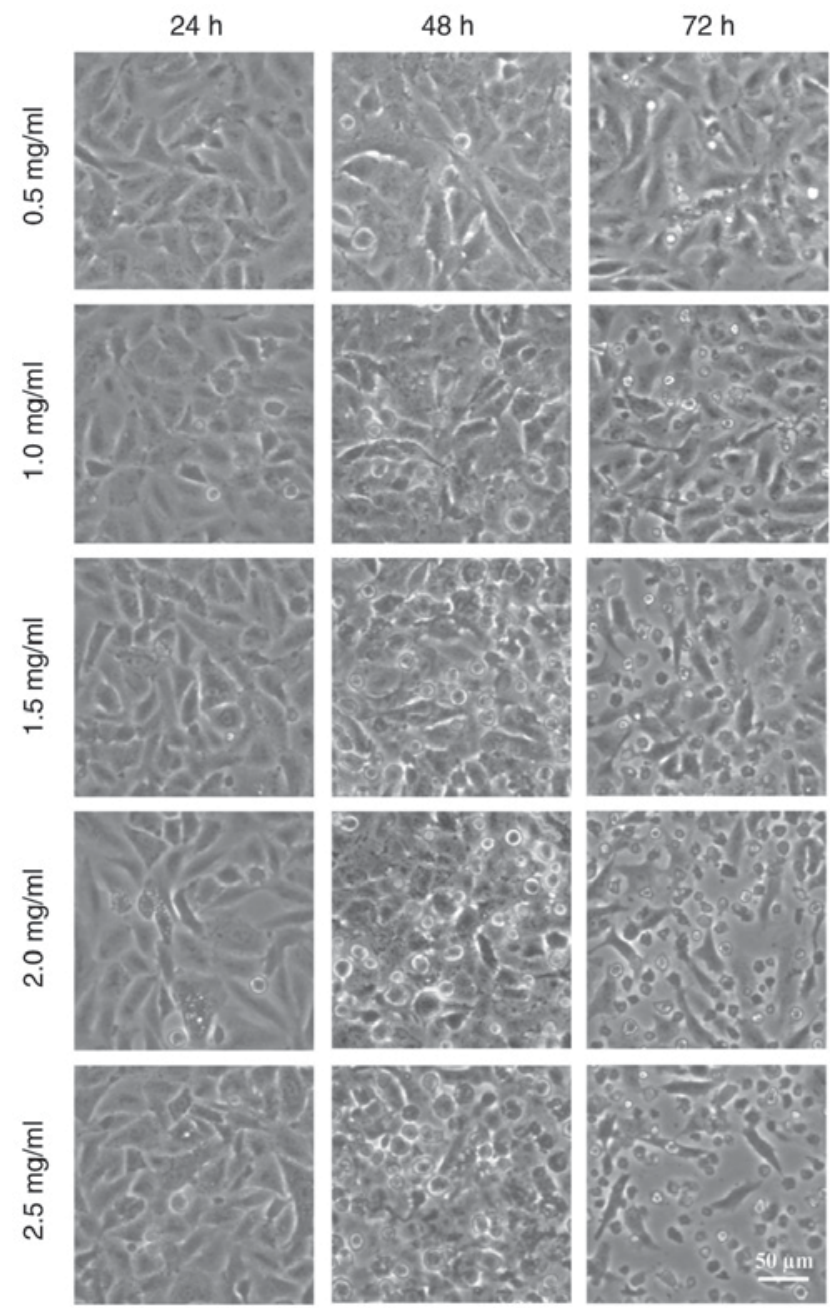

Figure 1. Morphological alterations of oxymatrine-treated A549 cells. Cells were incubated with oxymatrine $(0.5-2.5 \mathrm{mg} / \mathrm{l})$ over differing time-periods. Images were captured under an inverted microscope (scale bar, $50 \mu \mathrm{m}$ ).

(version 19.0; IBM Corp., Armonk, NY, USA) and expressed as the mean \pm standard error. $\mathrm{P}<0.05$ was considered to indicate a statistically significant difference.

\section{Results}

Oxymatrine induces an anti-proliferative effect in A549 cells. Microscope observation analysis indicated that A549 cells became rounded and granular following treatment with oxymatrine at different concentrations (Fig. 1). At $24 \mathrm{~h}$, the treated cells did not appear to exhibit any properties characteristic of apoptosis. However, over the increasing time period, the treated A549 cells detached from the monolayer and floated in the cell medium, and this effect appeared to be enhanced with increased concentrations of oxymatrine.

Furthermore, MTT analysis was applied to identify the A549 cell viability following treatment. The experiments demonstrated that oxymatrine inhibited A549 cell growth in a time- and dose-dependent manner (Fig. 2). When the treatment concentration of oxymatrine increased, the A549 cell viability continuously decreased. Overall, the aforementioned results suggested that oxymatrine had an anti-proliferative effect on the A549 lung cancer cells.

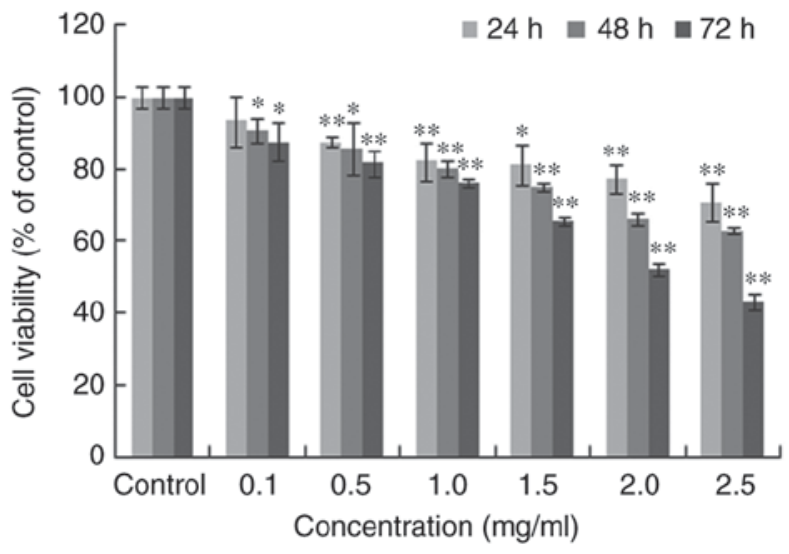

Figure 2. Effects of oxymatrine on viability of A549 cells. A549 cells were exposed to various concentrations of oxymatrine over differing time-periods and an MTT assay was applied to detect the cell viability. Data are presented as the mean \pm standard error of the mean $(n=3)$. ${ }^{*} \mathrm{P}<0.05$ and ${ }^{* *} \mathrm{P}<0.01$ vs. the respective control group.

Oxymatrine induces apoptosis in A549 cells. To further identify the apoptosis mechanism of A549 induced by oxymatrine, Hoechst/PI dual-staining assay was employed to detect possible cell nuclei alterations. Generally, Hoechst enters into the normal cytomembrane and stains the nucleolus blue, whereas PI is a nucleic acid dye that only has access to the damaged cytomembrane and stains nuclei red, therefore is often used for assessment of the late apoptotic cells. Hoechst 33342 staining exhibited apparent nuclear fragmentation at 48 h (Fig. 3A), whereas the nuclei of control cells remained intact. The red fluorescence of PI was additionally detected, suggesting that oxymatrine induce apoptosis of A549 cells.

Given that apoptosis is often caspase-dependent, the caspase inhibitor z-VAD-FMK was used to pre-incubate the A549 cells, and then fluorescence observation analysis revealed that z-VAD-FMK, in part, inhibited the fragmentation of A549 cell nuclei (Fig. 3B). This suggested that the oxymatrine-induced apoptosis of A549 cells was caspase-dependent.

Further identification of oxymatrine-induced apoptosis in A549 cells. Fluorescence microscopy analysis was used to study apoptosis of A549 cells following oxymatrine treatment. In addition to this, TUNEL and flow cytometry analysis were applied to further verify the apoptotic characteristics of A549 cells. TUNEL analysis demonstrated that the apoptotic cells contracted and the nuclei appeared navy and the perinuclei brown in the oxymatrine-treated group, whereas the positive signals were not detected in control cells (Fig. 4).

Furthermore, as a lipophilic and cationic dye, JC-1 aggregates locate in areas with high mitochondrial membrane potential $(\Delta \Psi \mathrm{m})$ and emits red fluorescence, and JC-1 monomers emit green fluorescence, and locate in low $\Delta \Psi \mathrm{m}$ cell areas. The ratio of red/green fluorescence acts as a marker of $\Delta \Psi \mathrm{m}$ alterations in cells (18). In the present study, JC-1 staining revealed a decrease of red/green ratio following treatment with oxymatrine for 12, 48 and $72 \mathrm{~h}$, respectively (Fig. 5A). The decrease of $\Delta \Psi \mathrm{m}$ further verified that oxymatrine induced A549 cell apoptosis.

In addition, to determine the cause of apoptosis, flow cytometry analysis was applied to study the cell arrest of 
A Hoechst
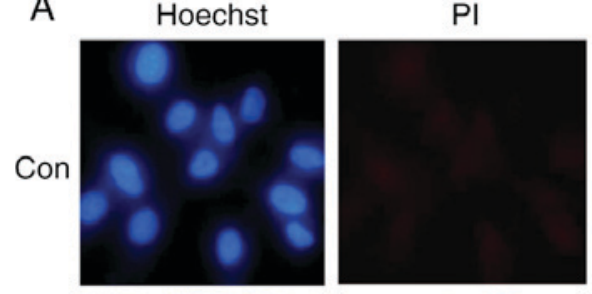

48
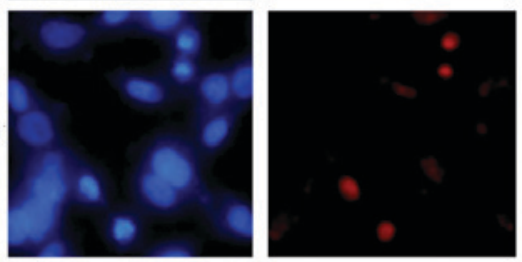

B
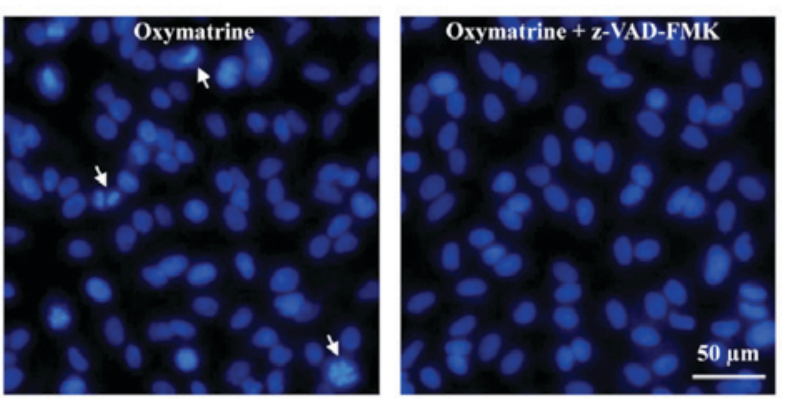

Figure 3. Detection of apoptosis in oxymatrine-treated A549 cells (A) Treated cells were stained with Hoechst 33342 and PI. Con and $48 \mathrm{~h}$ represent the blank control group and the oxymatrine-treated cell sample respectively. (B) Presence of z-VAD-FMK blocked nuclei fragmentation of oxymatrine-treated A549 cells. All images were captured by a fluorescence microscope (Scale bar, $50 \mu \mathrm{m}$ ). PI, propidium iodide.
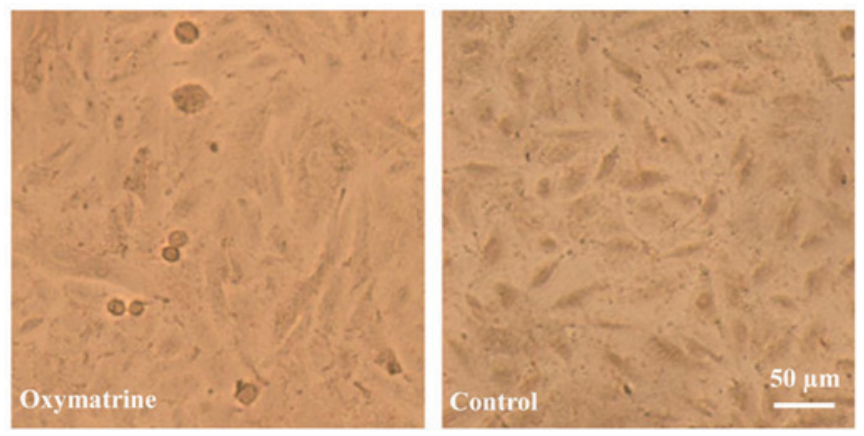

Figure 4. Terminal deoxynucleotidyl transferase dUTP nick end labeling analysis of oxymatrine-treated A549 cells. The navy brown staining signals revealed the apoptotic cells (left). Untreated cells were used as control (right). Scale bar, $50 \mu \mathrm{m}$.

oxymatrine-treated A549 cells. The results suggested that the DNA synthesis decreased in a time dependent manner, and the number of G0/G1 cells increased prior to $48 \mathrm{~h}$ (Fig. 5B). Compared with control cells, treated groups at different time-points all revealed $\mathrm{S}$ phase arrest. The above mentioned experiments verified that oxymatrine resulted in A549 cell arrest and induced apoptosis. Future studies should focus on the specific molecular apoptotic mechanism of A549 cells.

Oxymatrine-induced apoptosis in A549 cells is caspase-dependent. To further investigate whether the apoptosis induced by oxymatrine was caspase-dependent or not,

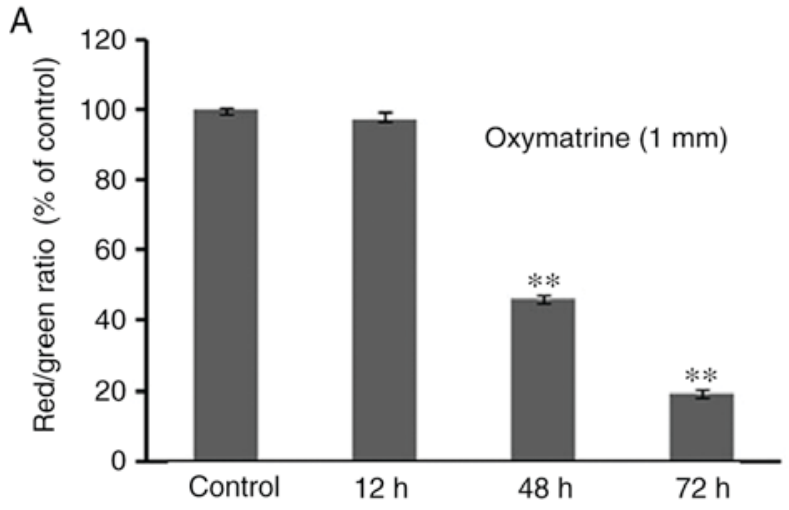

B
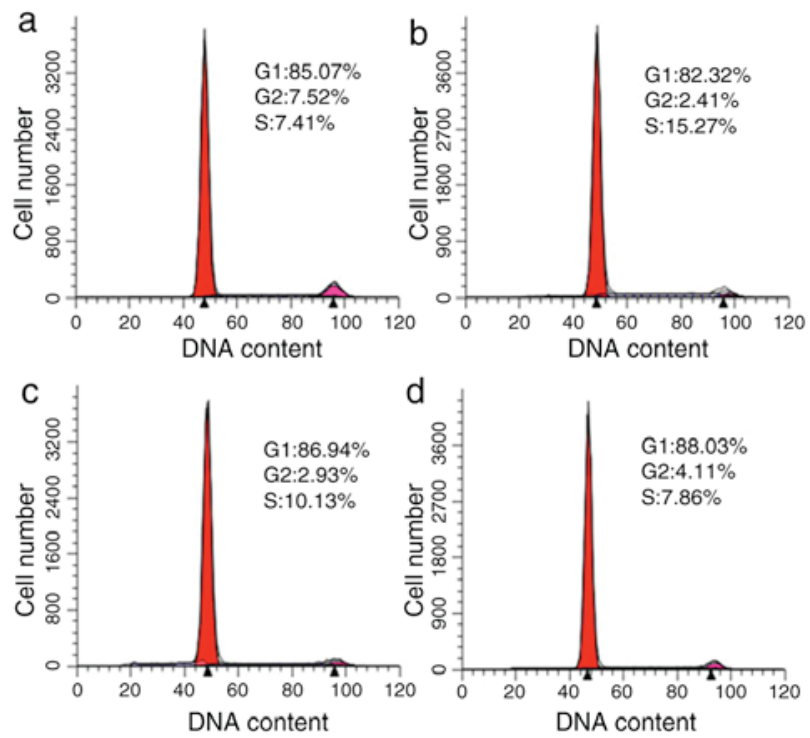

Figure 5. Effects of oxymatrine-induced apoptosis detected via JC-1 staining and flow cytometric analysis. (A) A549 cell mitochondrial membrane potential $(\Delta \Psi \mathrm{m})$ detection following treatment with oxymatrine at 12,48 and $72 \mathrm{~h}$, respectively. (B) Flow cytometry analysis of oxymatrine-treated A549 cells for (a) control, (b) 24, (c) 48 and (d) 72 h. ${ }^{* *} \mathrm{P}<0.01$ vs. the control.

the oxymatrine-treated ( 48 and $72 \mathrm{~h}$ ) or untreated control A549 cells were harvested by scraping. The supernatants of these cell lysates were detected for the activities of several key caspases in the process of apoptosis. The results demonstrated that the absorbance $\left(\mathrm{OD}_{405 \mathrm{~nm}}, \mathrm{n}=3\right)$ of oxymatrine-treated cell supernatants all increased compared with the control cells $(\mathrm{P}<0.05$; Fig. 6) which suggested that caspases-3, 8 and 9 were activated following oxymatrine treatment in A549 cells, and the induced apoptosis was caspase-dependent.

Oxymatrine inhibits migration of A549 cells. Due to their proliferative abilities, a further property of malignant tumor cells is the ability to migrate (19). The present study performed the wounding healing assay to examine the anti-migratory abilities of oxymatrine. The results demonstrated that following treatment with oxymatrine $(1.5$ and $2.0 \mathrm{mg} / \mathrm{ml})$ for different time periods, a decreased number of A549 cells migrated to the blank field (Fig. 7A). Increased concentrations of oxymatrine resulted in a smaller migrated cell number ratio (Fig. 7B). This suggested that oxymatrine inhibited migration of A549 cells. Future investigations should focus on factors involved in the migration of A549 cells following oxymatrine administration. 

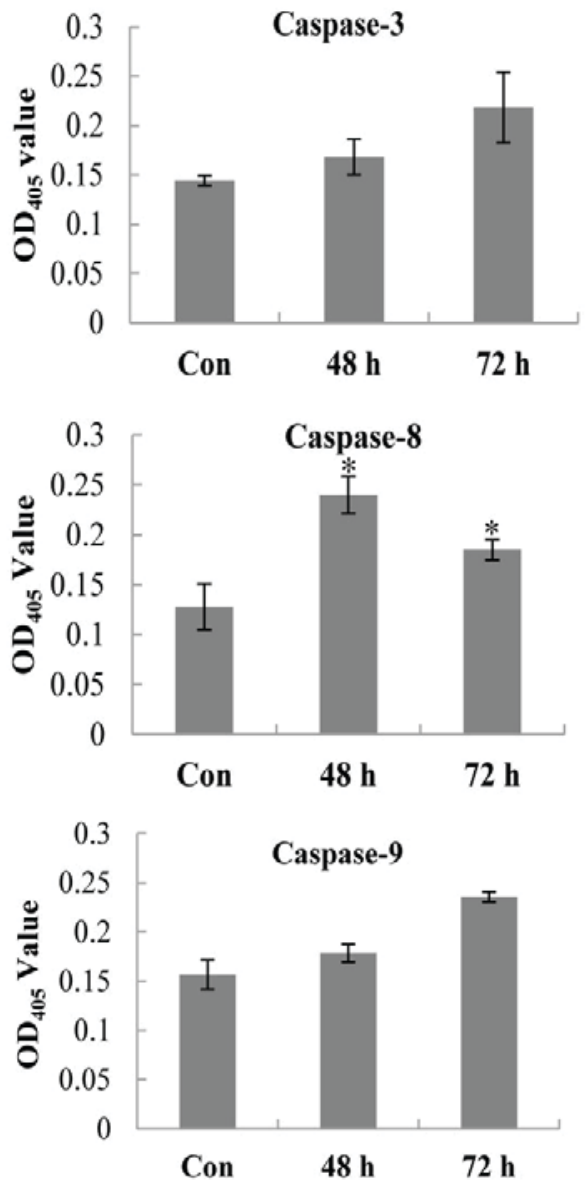

Figure 6. Activity detection of caspase-3, 8 and 9 of oxymatrine-treated A549 cells at 48 and $72 \mathrm{~h}$, respectively. Compared with control groups, the caspase cascade pathway was activated in the treated cells $(n=3)$. OD, optical density; Con, control. ${ }^{*} \mathrm{P}<0.05$ vs. the control group.

\section{Discussion}

The present study firstly applied traditional techniques, including light and fluorescence microscope observation, MTT analysis and TUNEL assay to detect the apoptotic characteristics of A549 cells following oxymatrine treatment for different time-periods, verifying that oxymatrine inhibited proliferation of A549 cells by inducing cell apoptosis. Furthermore, JC-1 staining was applied to monitor the decrease of $\Delta \Psi \mathrm{m}$ in oxymatrine-induced A549 cell apoptosis. Flow cytometry was used to analyze the cell arrest of A549 cells following oxymatrine treatment. The results of the aforementioned experiments demonstrated an apoptotic effect of oxymatrine in A549 cells. Finally, the caspase 3, 8 and 9 activities of oxymatrine-treated A549 cells were detected, suggesting that all caspases were activated following oxymatrine treatment at differing concentrations. Caspases (particularly caspase-3) are critical in regulating apoptosis and inflammation and caspase-dependent cell apoptosis primarily results from extrinsic and intrinsic apoptotic pathways. Caspase-8, caspase- 9 and caspase-12 are the specific caspases in the extrinsic and intrinsic apoptotic pathways (20). The present study currently analyzed the activities of the aforementioned three caspases in oxymatrine-treated A549 cells, and results indicated increased activity for all, compared with controls.

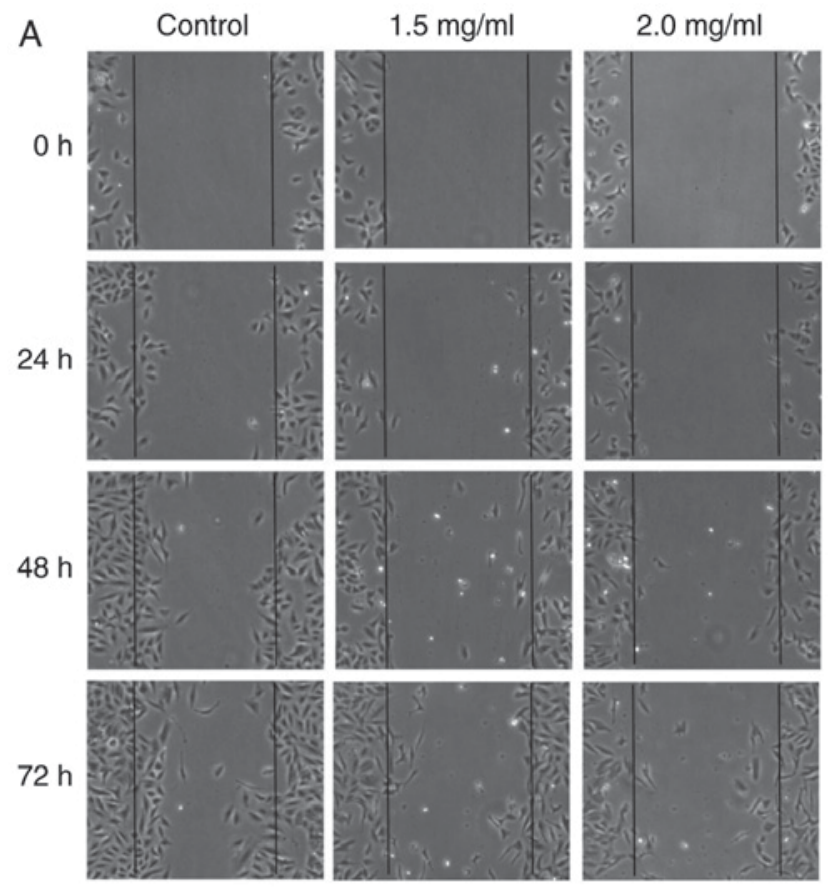

B

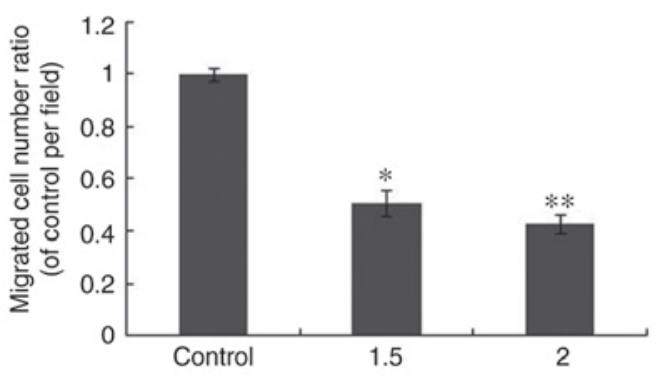

Figure 7. Oxymatrine inhibits A549 cell migration. (A) Wound healing assay examined the anti-migratory ability of oxymatrine (magnification $\mathrm{x} 400$ ). (B) Statistical analysis of migrated cell number ratio following treatment with oxymatrine at $72 \mathrm{~h}(\mathrm{n}=3) .{ }^{*} \mathrm{P}<0.05$ and ${ }^{* *} \mathrm{P}<0.01$ vs. the control group.

It was therefore suggested that oxymatrine induced apoptosis of A549 cells via extrinsic and intrinsic pathways. The present study firstly verified the apoptotic modes of oxymatrine-induced A549 lung cancer, and following work should focus on the specific apoptosis-associated cellular proteins, or other factors involved in the anti-proliferative process.

Cell migration during cancer progression determines the capacity of tumor cells to escape from the primary tumors and invade adjacent tissues to finally form metastases, and cancer cells modify their migration mechanisms in response to different conditions, which makes cancer therapeutics designed to target adhesion receptors or proteases in slowing tumor progression in clinical trials inefficient (21). Therefore, research into the cellular and molecular basis of these different migration programs and developing novel chemical drugs may lead to novel potential treatment strategies. The present study applied the wound healing assay to assess the effect of oxymatrine in A549 cells, and it was demonstrated that oxymatrine inhibited the migration of A549 cells in vitro. However, whether its anti-migration effect in lung cancer cells exists in vivo is still not known, and future work should be carried out to investigate the specific mechanism underlying A549 cell dissemination. 
Oxymatrine is a traditional Chinese medicine that has been demonstrated to induce anti-cancer effects (1). The present study revealed that oxymatrine inhibited proliferation of A549 cells. One previous study also suggested that oxymatrine inhibits cell proliferation by inducing apoptosis in human lung cancer A549 cells, however specific apoptosis pathways remain to be elucidated (22).

In conclusion, the present study further demonstrated that oxymatrine could induce A549 lung cancer cell apoptosis via the extrinsic and intrinsic pathways.

\section{Acknowledgements}

The present study was supported, in part, by the Science and Technology Planning Project of Henan Province, China (grant no. 172102410082), the Foundation of Henan Educational Committee, China (grant no. 16A180027), the Training Program for Youth Backbone Teacher of Henan University of Technology (grant no. 001170) and the Foundation for University Key Teacher by the Henan Province, China (grant no. 2015GGJS-041).

\section{References}

1. Liu Y, Xu Y, Ji W, Li X, Sun B, Gao Q and Su C: Anti-tumor activities of matrine and oxymatrine: Literature review. Tumor Biol 35: 5111-5119, 2014.

2. Wang CY, Bai XY and Wang CH: Traditional Chinese medicine: A treasured natural resource of anticancer drug research and development. Am J Chin Med 42: 543-559, 2014.

3. He M, Wu Y, Wang M, Chen W and Jiang J: Meta-analysis of the clinical value of oxymatrine on sustained virological response in chronic hepatitis B. Ann Hepatol 15: 482-491, 2016.

4. Liu Y, Bi T, Wang Z, Wu G, Qian L, Gao Q and Shen G: Oxymatrine synergistically enhances antitumor activity of oxaliplatin in colon carcinoma through PI3K/AKT/mTOR pathway. Apoptosis 21: 1398-1407, 2016.

5. Li J, Jiang K and Zhao F: Oxymatrine suppresses proliferation and facilitates apoptosis of human ovarian cancer cells through upregulating microRNA-29b and downregulating matrix metalloproteinase-2 expression. Mol Med Rep 12: 5369-5374, 2015.

6. Liu Y, Bi T, Dai W, Wang G, Qian L, Gao Q and Shen G: Effects of oxymatrine on the proliferation and apoptosis of human hepatoma carcinoma cells. Technol Cancer Res Treat 15: 487-497, 2016.
7. Guo B, Zhang T, Su J, Wang K and Li X: Oxymatrine targets EGFR(p-Tyr845) and inhibits EGFR-related signaling pathways to suppress the proliferation and invasion of gastric cancer cells. Cancer Chemother Pharmacol 75: 353-363, 2015.

8. Wu C, Huang W, Guo Y, Xia P, Sun X, Pan X and Hu W: Oxymatrine inhibits the proliferation of prostate cancer cells in vitro and in vivo. Mol Med Rep 11: 4129-4134, 2015.

9. Field JK and Duffy SW: Lung cancer screening: The way forward. Br J Cancer 99: 557-562, 2008.

10. Pore MM, Hiltermann TJ and Kruyt FA: Targeting apoptosis pathways in lung cancer. Cancer Lett 332: 359-368, 2013.

11. Heist RS and Engelman JA: SnapShot: Non-small cell lung cancer. Cancer Cell 21: 448, 2012.

12. Azzoli CG, Temin S, Aliff T, Baker S Jr, Brahmer J, Johnson DH, Laskin JL, Masters G, Milton D, Nordquist L, et al: 2011 focused update of 2009 American Society of Clinical Oncology clinical practice guideline update on chemotherapy for stage IV non-small-cell lung cancer. J Clin Oncol 29: 3825-3831, 2011.

13. Mehta HJ, Patel V and Sadikot RT: Curcumin and lung cancer--a review. Target Oncol 9: 295-310, 2014.

14. Feng Y, Zhou J and Jiang Y: Resveratrol in lung cancer-a systematic review. J BUON 21: 950-953, 2016.

15. Zhou GZ, Xu SL, Sun GC and Chen XB: Novel curcumin analogue IHCH exhibits potent anti-proliferative effects by inducing autophagy in A549 lung cancer cells. Mol Med Rep 10: 441-446, 2014.

16. Zhou GZ, Zhang SN, Zhang L, Sun GC and Chen XB: A synthetic curcumin derivative hydrazinobenzoylcurcumin induces autophagy in A549 lung cancer cells. Pharm Biol 52: 111-116, 2014.

17. Zhou GZ, Cao FK and Du SW: The apoptotic pathways in the curcumin analog MHMD-induced lung cancer cell death and the essential role of actin polymerization during apoptosis. Biomed Pharmacoth 71: 128-134, 2015.

18. Gamal-Eldeen AM,Hamdy NA, Abdel-AzizHA,El-Hussieny EA and Fakhr IM: Induction of intrinsic apoptosis pathway in colon cancer HCT-116 cells by novel 2-substituted-5,6,7,8-tetrahydronaphthalene derivatives. Eur J Med Chem 77: 323-333, 2014.

19. Polacheck WJ, Zervantonakis IK and Kamm RD: Tumor cell migration in complex microenvironments. Cell Mol Life Sci 70: 1335-1356, 2013

20. Fulda S and Debatin KM: Extrinsic versus intrinsic apoptosis pathways in anticancer chemotherapy. Oncogene 25: 4798-4811, 2006.

21. Friedl $\mathrm{P}$ and Wolf K: Tumor-cell invasion and migration: Diversity and escape mechanisms. Nat Rev Cancer 3: 362-374, 2003.

22. Wang B, Han Q and Zhu Y: Oxymatrine inhibited cell proliferation by inducing apoptosis in human lung cancer A549 cells. Biomed Mater Eng 26 (Suppl 1): S165-S172, 2015. 